Network Working Group

Request for Comments: 4525

Category: Informational
K. Zeilenga

OpenLDAP Foundation

June 2006

\title{
Lightweight Directory Access Protocol (LDAP)
}

Modify-Increment Extension

Status of This Memo

This memo provides information for the Internet community. It does not specify an Internet standard of any kind. Distribution of this memo is unlimited.

Copyright Notice

Copyright (C) The Internet Society (2006).

Abstract

This document describes an extension to the Lightweight Directory Access Protocol (LDAP) Modify operation to support an increment capability. This extension is useful in provisioning applications, especially when combined with the assertion control and/or the preread or post-read control extension.

Table of Contents

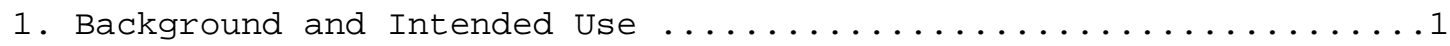

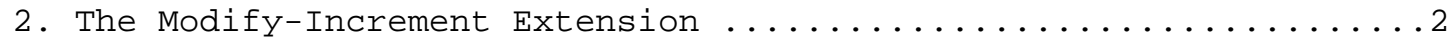

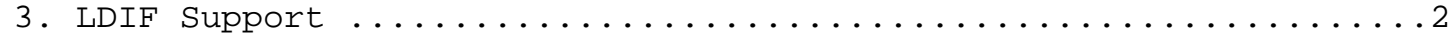

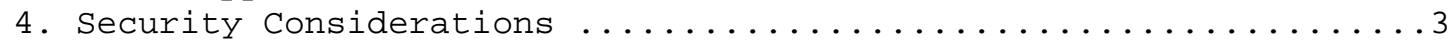

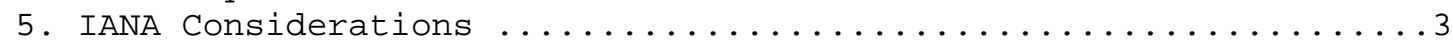

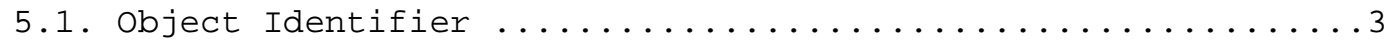

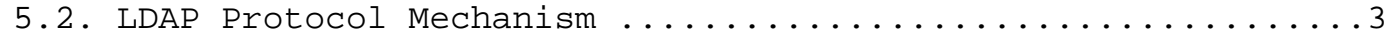

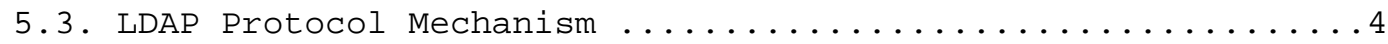

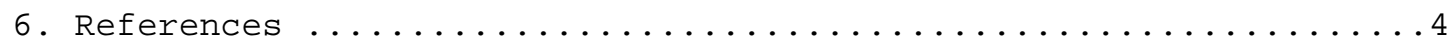

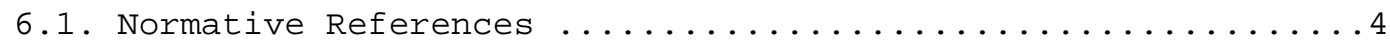

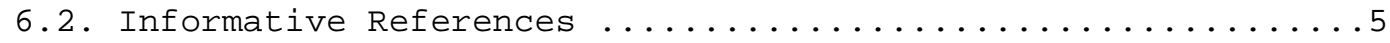

1. Background and Intended Use

The Lightweight Directory Access Protocol (LDAP) [RFC4510] does not currently provide an operation to increment values of an attribute. A client must read the values of the attribute and then modify those values to increment them by the desired amount. As the values may be updated by other clients between this add and modify, the client must 
be careful to construct the modify request so that it fails in this case, and upon failure, to re-read the values and construct a new modify request.

This document extends the LDAP Modify Operation [RFC4511] to support an increment values capability. This feature is intended to be used with either the LDAP pre-read or post-read control extensions [RFC4527]. This feature may also be used with the LDAP assertion control extension [RFC4528] to provide test-and-increment

functionality.

In this document key words "MUST", "MUST NOT", "REQUIRED", "SHALL", "SHALL NOT", "SHOULD", "SHOULD NOT", "RECOMMENDED", "MAY", and "OPTIONAL" are to be interpreted as described in BCP 14 [RFC2119].

\section{The Modify-Increment Extension}

This document extends the LDAP Modify request to support a increment values capability. Implementations of this extension SHALL support an additional ModifyRequest operation enumeration value increment (3), as described herein. Implementations not supporting this extension will treat this value as they would an unlisted value, e.g., as a protocol error.

The increment (3) operation value specifies that an increment values modification is requested. All existing values of the modification attribute are to be incremented by the listed value. The modification attribute must be appropriate for the request (e.g., it must have INTEGER or other increment-able values), and the modification must provide one and only one value. If the attribute is not appropriate for the request, a constraintViolation or other appropriate error is to be returned. If multiple values are provided, a protocolerror is to be returned.

Servers supporting this feature SHOULD publish the object identifier (OID) 1.3.6.1.1.14 as a value of the 'supportedFeatures' [RFC4512] attribute in the root DSE. Clients supporting this feature SHOULD NOT use the feature unless they know the server supports it.

3. LDIF Support

To represent Modify-Increment requests in LDAP Data Interchange Format [RFC2849], the ABNF [RFC4234] production <mod-spec> is extended as follows:

mod-spec =/ "increment:" FILL AttributeDescription SEP attrval-spec "-" SEP 
For example,

\# Increment uidNumber

dn: $c n=m a x-a s s i g n e d$ uidNumber $, d c=e x a m p l e, d c=c o m$

changetype: modify

increment: uidNumber

uidNumber: 1

$-$

This LDIF fragment represents a Modify request to increment the value(s) of uidNumber by 1 .

4. Security Considerations

General LDAP security considerations [RFC4510], as well as those specific to the LDAP Modify [RFC4511], apply to this Modify-Increment extension. Beyond these considerations, it is noted that

introduction of this extension should reduce application complexity (by providing one operation for what presently requires multiple operations) and, hence, it may aid in the production of correct and secure implementations.

5. IANA Considerations

Registration of the following values [RFC4520] have been completed.

5.1. Object Identifier

The IANA has assigned an LDAP Object Identifier to identify the LDAP Modify-Increment feature, as defined in this document.

Subject: Request for LDAP Object Identifier Registration

Person \& email address to contact for further information: Kurt Zeilenga <kurt@openLDAP.org>

Specification: RFC 4525

Author/Change Controller: Author

Comments : Identifies the LDAP Modify-Increment feature

5.2. LDAP Protocol Mechanism

The following LDAP Protocol Mechanism has been registered.

Subject: Request for LDAP Protocol Mechanism Registration Object Identifier: 1.3.6.1.1.14

Description: Modify-Increment

Person \& email address to contact for further information:

Kurt Zeilenga <kurt@openldap.org> 


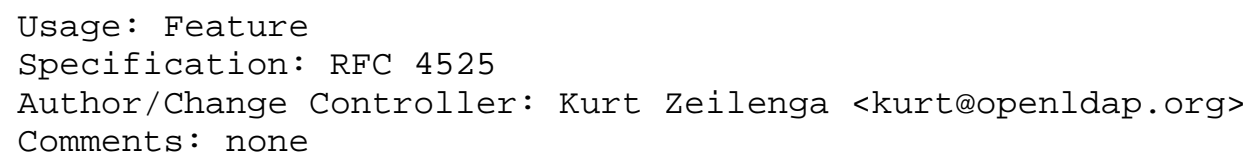

6. References

6.1. Normative References

[RFC2119] Bradner, S., "Key words for use in RFCs to Indicate Requirement Levels", BCP 14, RFC 2119, March 1997.

[RFC4234] Crocker, D. and P. Overell, "Augmented BNF for Syntax Specifications: ABNF", RFC 4234, October 2005.

[RFC2849] Good, G., "The LDAP Data Interchange Format (LDIF) Technical Specification", RFC 2849, June 2000.

[RFC4510] Zeilenga, K., Ed., "Lightweight Directory Access Protocol (LDAP): Technical Specification Road Map", RFC 4510, June 2006 .

[RFC4511] Sermersheim, J., Ed., "Lightweight Directory Access Protocol (LDAP): The Protocol", RFC 4511, June 2006.

[RFC4512] Zeilenga, K., "Lightweight Directory Access Protocol (LDAP): Directory Information Models", RFC 4512, June 2006 . 
6.2. Informative References

[RFC4520] Zeilenga, K., "Internet Assigned Numbers Authority (IANA) Considerations for the Lightweight Directory Access Protocol (LDAP)", BCP 64, RFC 4520, June 2006.

[RFC4527] Zeilenga, K., "Lightweight Directory Access Protocol (LDAP) Read Entry Controls", RFC 4527, June 2006.

[RFC4528] Zeilenga, K., "Lightweight Directory Access Protocol (LDAP) Assertion Control", RFC 4528, June 2006.

Author's Address

Kurt D. Zeilenga

OpenLDAP Foundation

EMail: Kurt@openLDAP.org 
Full Copyright statement

Copyright (C) The Internet Society (2006).

This document is subject to the rights, licenses and restrictions contained in BCP 78, and except as set forth therein, the authors retain all their rights.

This document and the information contained herein are provided on an "AS IS" basis and THE CONTRIBUTOR, THE ORGANIZATION HE/SHE REPRESENTS OR IS SPONSORED BY (IF ANY), THE INTERNET SOCIETY AND THE INTERNET ENGINEERING TASK FORCE DISCLAIM ALL WARRANTIES, EXPRESS OR IMPLIED, INCLUDING BUT NOT LIMITED TO ANY WARRANTY THAT THE USE OF THE INFORMATION HEREIN WILL NOT INERINGE ANY RIGHTS OR ANY IMPLIED WARRANTIES OF MERCHANTABILITY OR FITNESS FOR A PARTICULAR PURPOSE.

Intellectual Property

The IETF takes no position regarding the validity or scope of any Intellectual Property Rights or other rights that might be claimed to pertain to the implementation or use of the technology described in this document or the extent to which any license under such rights might or might not be available; nor does it represent that it has made any independent effort to identify any such rights. Information on the procedures with respect to rights in RFC documents can be found in BCP 78 and BCP 79 .

Copies of IPR disclosures made to the IETF Secretariat and any assurances of licenses to be made available, or the result of an attempt made to obtain a general license or permission for the use of such proprietary rights by implementers or users of this specification can be obtained from the IETF on-line IPR repository at http://www.ietf.org/ipr.

The IETF invites any interested party to bring to its attention any copyrights, patents or patent applications, or other proprietary rights that may cover technology that may be required to implement this standard. Please address the information to the IETF at ietf-ipreietf.org.

Acknowledgement

Funding for the RFC Editor function is provided by the IETF Administrative Support Activity (IASA). 